\title{
LAS IDEAS DE LOS PROFESORES DE CIENCIAS SOBRE LA FORMACIÓN DOCENTE
}

\author{
MARTÍNEZ LOSADA, C., GARCÍA BARROS, S. y MONDELO ALONSO, M. \\ Departamento de Pedagoxía e Didáctica das Ciencias Experimentais. EU de Formación del Profesorado de \\ EGB. Universidad de La Coruña. Paseo de Ronda, 43. 15011 La Coruña.
}

\section{SUMMARY}

In this paper we investigate novel teachers' notions and practice regarding their conceptions about the need to receive a specific teaching formation, and the most suitable way to achieve this formation.

\section{INTRODUCCIÓN}

Los profesores de Ciencias somos conscientes de que la enseñanza de esta disciplina no es una tarea fácil, constatándose un alto fracaso escolar en la misma. En las últimas décadas se ha observado un importante desarrollo de la actividad investigadora en Didáctica de las Ciencias, cuyo objetivo, entre otros, ha sido promover un cambio metodológico y una fundamentación teórica de su enseñanza, perfilando cuáles han de ser las líneas generales de la alfabetización científica en los diferentes niveles de la educación obligatoria. En este sentido cabe destacar la nueva filosofía que marca la selección de objetivos/contenidos en los currículos de Ciencias de la enseñanza elemental y media: "Ciencia para todos» y ampliación de los ámbitos de los objetivos, incluyendo, además de los referidos a conceptos, aquéllos referidos a procesos, actitudes, contexto y los metacientíficos (Gutiérrez et al. 1990)

Esta nueva visión de la educación científica demanda necesariamente un nuevo perfil de profesor; tiene que estar mejor preparado profesionalmente para abordar con éxito los actuales retos de la enseñanza de las Ciencias en cuanto a «qué Ciencias enseñar» y "cómo hacerlo». El profesor de Ciencias, además de realizar labores técnicas, ha de elaborar materiales curriculares adaptados a su aula y a su disciplina. Para ello se requiere indudablemente:

a) Un conocimiento teórico profundo de la materia a enseñar (Tobin y Espinet 1989), aunque no circunscrito exclusivamente al ámbito de los conceptos, leyes, teorías, etc., sino más amplio y «cultural» del que desgraciadamente se ha despreocupado la enseñanza tradicio- nal de las Ciencias en los distintos niveles educativos. Nos referimos a aspectos tales como el conocimiento de la evolución y construcción del conocimiento científico (Matthews 1990, Otero 1989, Saltiel y Viennot 1985), de las relaciones Ciencia/Tecnología/Sociedad (Solbes y Vilches 1989, Jiménez y Otero 1990), de los nuevos descubrimientos científicos que nos permiten a los profesores transmitir una visión dimámica de la Ciencia (Gil 1991).

b) Un conocimiento del cuerpo teórico que configura lo que actualmente entendemos por Didáctica de las Ciencias. Ésta ha de convertirse «en el núcleo vertebrador de la formación docente) (Dumas-Carré et al. 1990, Gil 1991, documento de trabajo), y por ello tiene la misión, entre otras, de asociar los aspectos psicopedagógicos generales a los problemas más específicos que encierra la enseñanza de las Ciencias.

c) Una introducción en la investigación didáctica. La investigacion, al igual que ocurre en otras profesiones, ha de nutrir la propia actividad docente cotidiana (Verma y Beard 1981). El profesor de Ciencias debe aproximarse a la investigación en este campo, tanto en los niveles de formación inicial como en su formación continua (Lawson 1986, Penick y Yager 1986), si realmente se pretende que se transforme en un profesor innovador y crítico que lleve a cabo las modificaciones y adecuaciones curriculares que los tiempos van demandando.

Todo lo expuesto nos conduce necesariamente a reivindicar una buena formación profesional de los profesores de los distintos niveles. Hasta hace poco, Ia filosofía que 
ha marcado tal formación se basó en la idea ingenua de que ser profesor es una profesión eminentemente «vocacional» y en que el profesor «nace», al tener éste unas características personales idóneas para la función docente, previo conocimiento, lógicamente, de la o las disciplinas a impartir. Teniendo en cuenta esto, las personas «idóneas", "nacidas docentes", van adquiriendo mayor experiencia a través de lo que Zeichner (1983) llama, y critica a la vez, paradigma «tradicional-artesanal», según el cual el profesor, por meros procesos de ensayo-error. aprenderá y mejorará su actividad profesional. De acuerdo con el mismo autor, a esta filosof́a se enfrenta otra que entiende que el docente ha de sufrir un proceso de formación científico/didáctico conjunto. A su vez, en la citada formación se destaca la realización de prácticas que permitan al profesor reflexionar críticamente sobre sus planteamientos didácticos, su propia actuación en el aula, etc., introduciéndolo en la investigación-acción (Calderhead 1989).

A la luz de esta última filosofía, y siguiendo el modelo constructivista de la enseñanza de las Ciencias, en la formación de profesores resultará imprescindible que los nuevos docentes evidencien, cuestionen y analicen sus propias ideas sobre qué y cómo enseñar Ciencias. De esta forma se pondrá de manifiesto el «pensamiento docente del sentido común» (Gené y Gil 1987, Gil et al. 1991), paso inicial para promover el cambio conceptual y metodológico indicado anteriormente.

Así mismo es esencial evidenciar sus opiniones sobre la influencia de la formación docente en la calidad de la enseñanza y, en definitiva, en la alfabetización científica de los estudiantes. En este sentido es frecuente que los docentes atribuyan a factores externos a la propia docencia (capacidad intelectual de los alumnos, razones socioculturales, falta de medios económicos y materiales, masificación de las aulas...) la responsabilidad del fracasoescolar, desestimando la formación inicial y permanente del profesorado como un importante factor a tener en cuenta.

Por todo lo expuesto, en este trabajo pretendemos aportar datos sobre el grado de concienciación de los distintos grupos de profesores (en ejercicio y en formación) con respecto a la necesidad de recibir una formación profe. sional específica. Para ello estudiaremos hasta qué punto ésta es considerada como un factor influyente en la consecución de los objetivos actualmente planteados en la enseñanza de las Ciencias en los niveles obligatorios. Finalmente analizaremos cuáles son las vías más idóneas de formación a juício de los distintos grupos de profesores y la influencia del «ambiente» en este aspecto.

\section{MÉTODO}

Se han realizado encuestas a 35 alumnos de $3^{2}$ de la Escuela Universitaria de Profesorado de EGB de La Coruña de la especialidad de Ciencias (grupo A), a 35 alumnos del CAP de las especialidades de Ciencias (Biología y Química) (grupo B) y a 17 profesores en activo de EGB y BUP (grupo C).

El cuestionario incluye preguntas cerradas y abiertas (véase Anexo). Todos los grupos recibieron la misma encuesta. Sin embargo, una vez analizados los primeros resultados correspondientes al grupo A, consideramos conveniente hacer una mayor especificación de la opción $d$ en la pregunta 8. Mientras que al grupo $\mathrm{A}$ se le propuso únicamente valoración de la asistencia a «Cursos especializados de temas educativos», a los grupos B y $C$ se les presentaron dos opciones: «Asistencia a cursos de formación psicopedagógica» y «Asistencia a cursos de Didáctica de las Ciencias».

\section{RESULTADOS}

Tanto los profesores en ejercicio como los alumnos de la Escuela de Magisterio manifiestan mayoritariamente un alto grado de interés por la enseñanza de las Ciencias, según se desprende de la valoración de la pregunta 1 (más del $80 \%$ da una puntuación igual o mayor a 4). Sin embargo, únicamente el $54,3 \%$ de los alumnos del CAP manifiestan tal interés.

\section{Objetivos de la enseñanza de las ciencias en la educación obligatoria}

El análisis de la pregunta 2 nos permite identificar cuáles son los objetivos que, a juicio de los encuestados, ha de perseguir la enseñanza de las Ciencias en la educación obligatoria. En ella se incluyeron objetivos de contenidos sin ningún matiz (opción A); objetivos de contexto (Gutiérrez et al. 1990), es decir, objetivos de contenidos, pero matizando el interés que posee el conocimiento científico en el contexto cotidiano y su repercusión social y personal (opciones $\mathrm{B}$ y C); objetivos referidos a los procesos de la Ciencia (opción D) y objetivos de la dimensión metacientífica (opción $\mathrm{E}$ ).

En general los objetivos propuestos han sido altamente valorados por todos los grupos, si bien cabe destacar la escasa importancia mostrada hacia los objetivos del primer tipo (opción A de la encuesta); únicamente el $22,9 \%$ del grupo $\mathrm{A}$, el $37,0 \%$ del $\mathrm{B}$ y el $22,5 \%$ del $\mathrm{C}$ le adjudicaron una puntuación superior a 3 .

La priorizacion de objetivos extraída de las respuestas de los profesores en ejercicio y en formación se recoge

Tabla I

Priorización de los distintos tipos de objetivos que han de alcanzarse con la enseñanza de las ciencias en la educación obligatoria.

\section{GRUPO}

A

B

C

(1) $\mathrm{a}=$ objetivos de conocimiento de conceptos, teorías

b, $\mathrm{c}=$ objetivos de contexto

$d=$ objetivos de proceso

$e=$ objetivos metacientíficos 
en la tabla I. Los objetivos de proceso resultan prioritarios para los profesores en formación, mientras que los profesionales los relegan a penúltimo lugar, dándoles éstos mayor importancia a los objetivos de contexto. Los objetivos metacientíficos son valorados en segundo lugar por los futuros profesores de EGB y por los profesores en ejercicio, mientras que los alumnos del CAP los sitúan en tercer lugar.

\section{Causas que impiden que los estudiantes alcancen los objetivos deseados. Importancia otorgada a la formación docente}

Antes de abordar este tema nos pareció interesante estudiar cuáles son los objetivos reales que, a juicio de los profesores en formación y en ejercicio, llegan a alcanzar los estudiantes al finalizar la educación obligatoria (pregunta 3). Las respuestas no son sustancialmente diferentes entre los tres grupos encuestados y las podríamos resumir así:

a) Ideas muy generales sobre teorías y conceptos cien. tíficos.

b) Conocimientos exclusivamente memorísticos, mal comprendidos.

c) La enseñanza de las Ciencias les permite adaptarse al sistema educativo actual (se aprende a realizar pruebas de evaluación con éxito), lo que les capacita para acceder a estudios superiores (adquirir un título).

Otras respuestas mucho menos numerosas apuntan a aprender a manejar bibliografía y a promover una actitud crítica.

A juicio de los distintos grupos, «el desarrollo de actitudes positivas hacia la Ciencia y su aprendizaje» (pregunta 4) es un objetivo que, aunque interesante, no suelen alcanzar los estudiantes. Solamente 14 de los 79 sujetos encuestados $(17,7 \%)$ reconocen ei carácter motivador de la enseñanza de las Ciencias en los niveles obligatorios, mientras que $51(64,5 \%)$ lo niegan claramente. No existen diferencias entre los grupos, aunque los profesionaies son más pesimistas en este punto. Por otra parte, 6 alumnos de EU/CAP apuntan que el interés por las Ciencias depende del profesor; los profesores en ejercicio no apuntan esta posibilidad.

Un alto porcentaje de los encuestados estiman (pregunta 5) que la enseñanza de las Ciencias en los niveles elemental y medio no alcanza los objetivos que a su juicio deberían conseguirse (grupo $A, 74,1 \%$; grupo $B$, $62,9 \%$; grupo $\mathrm{C}, 82,3 \%$ ). Las condiciones que propiciarían la adquisición de tales objetivos, según opinión de los mismos (pregunta 6), son:

a) Reducción del número de alumnos por aula.

b) Reducción de los programas.

c) Realizar una enseñanza más activa, utilizando recursos experimentales y bibliográficos. d) Aproximar los temas de Ciencias a la realidad e intereses de los alumnos.

e) Mayor formación del profesorado.

f) Mayor atención a las capacidades de los alumnos y a aspectos psicopegagógicos en general.

Los puntos $a$ y $b$ fueron apuntados exclusivamente por la práctica totalidad de los profesores en ejercicio, el punto $f$ por cinco alumnos de la EU y los puntos $c, d$ y $e$ por todos los grupos. La utilización de estrategias de aprendizaje más activas (prácticas de campo/laboratorio) es la solución más apuntada por los distintos colectivos para alcanzar los objetivos deseados en la enseñanza de las Ciencias (grupo A, 57,1\%; grupo B, 51,8\%; grupo C, $29,4 \%$ ), siendo la menos señalada la $e$ (únicamente por uno o dos sujetos de cada colectivo).

\section{La formación del profesor de Ciencias}

Las opiniones de los profesores sobre las vías más idóneas de formación del docente no se pueden desligar de las correspondientes a los conocimientos teórico/ prácticos que ha de poseer el enseñante. Por tal motivo presentamos ambas en este apartado.

Con relación a este segundo aspecto propusimos las pregunta 7 de la encuesta. En ella se han incluido opciones de tipo técnico ("programar», "seleccionar contenidos», «evaluar alumnos»...) y otras que podríamos considerar más teóricas, que el profesor ha de conocer y tener en cuenta para desarrollar su actividad docente («analizar críticamente las nuevas tendencias en la enseñanza de las Ciencias», "comprender y aplicar las recomendaciones pedagógico/didácticas elaboradas por grupos de expertos»...). Las respuestas fueron muy similares en los tres grupos. Todos ellos dan una alta puntuación a las distintas opciones (prácticamente a todas se le otorgan valores superiores a 3 por más del $70 \%$ de los sujetos consultađos). Destacamos aquí la mayor consideración que los distintos colectivos mostraron hacia los quehaceres técnico-prácticos frente a aquéllos más generales o teóricos.

Tabla II

Opciones más valoradas en relación con la formación del profesor de Ciencias.

\begin{tabular}{|c|c|c|c|}
\hline OPCION & $\begin{array}{c}\text { GRUPO A } \\
\left(3^{2} \text { EU) }\right.\end{array}$ & $\begin{array}{c}\text { GRUPO B } \\
\text { (CAP) }\end{array}$ & $\begin{array}{c}\text { GRUPO C } \\
\text { (Prof.) }\end{array}$ \\
\hline Práctica docente & $65,7 \% *$ & $77,1 \%$ & $74,5 \%$ \\
\hline $\begin{array}{l}\text { Lectura de temas } \\
\text { cientifficos }\end{array}$ & $45,7 \%$ & $54,3 \%$ & $47,1 \%$ \\
\hline $\begin{array}{l}\text { Lectura de temas } \\
\text { didácticos especí- } \\
\text { fícos de Ciencias }\end{array}$ & $51,4 \%$ & $54,3 \%$ & $58,8 \%$ \\
\hline
\end{tabular}

* Los porcentajes se refieren al número de sujetos que otorgan una alta puntuacion $(<3)$ a las distintas opciones presentadas. 
Con el fin de estudiar más concretamente sus ideas acerca de la formación del profesorado de Ciencias, propusimos la pregunta 8. La opción más valorada de las que en ella se recogen fue «la observación cotidiana de su actuación en el aula y de la respuesta que a ella dan los alumnos», seguida de «la lectura de temas didácticos y científicos» (Tabla II).

Las otras opciones fueron menos consideradas. Solamente el 20\% de los estudiantes de Magisterio estima importante, para su formación, la asistencia a cursos de especialización. Los ítems más específicos referidos a este aspecto presentados a los profesores en ejercicio y a los alumnos del CAP muestran que ambos colectivos otorgan mayor importancia a los cursos de Didáctica de las Ciencias (valores superiores a 3: grupo B, el $37,1 \%$; grupo $\mathrm{C}$, el $29,4 \%$ ) que a los cursos más generales de formación psicopedagógica (valores superiores a 3 : grupo B, el 22,9\%; grupo C, el $5,8 \%$ ). A «la participación activa en la investigación educativa» y a «la asistencia a congresos" ies otorgaron puntuaciones superiores a 3 menos del $20 \%$ de los sujetos de los grupos A y C y alrededor del $30 \%$ de los del grupo $\mathrm{B}$.

\section{DISCUSIÓN}

En este trabajo nos encontramos con dos colectivos de docentes que manifiestan un alto interés por la enseñanza (los profesores de Ciencias en ejercicio y los futuros maestros), y otro (alumnos del CAP) cuyo interés es menor. Este último dato es coherente con nuestra propia experiencia en la formación del profesorado de Enserianza Media. A lo largo de la misma, hemos podido comprobar que la desmotivación de los alumnos del CAP del Área deCiencias es alta y que estos licenciados, salvo excepciones, suelen considerar la enseñanza como una salida profesional de segundo orden al no poder optar a otras posibilidades para las que sus estudios teóricamente les capacitan.

Con independencia del interés profesional de los distintos grupos encuestados, podemos considerar que entre ellos no existe una conciencia clara de la importancia que encierra la formación docente y de la necesidad de recibirla (Gil 1991). Esta afirmación la basamos en dos observaciones concretas derivadas de este estudio:

a) la formacion docente noes admitida generalmente como factor influyente en el éxito de la enseñanza científica;

b) la formación docente se concibe como un proceso autodidacta centrado en el ensayo-erxor, infuenciada por la docencia del sentido común (Gené y Gil 1987) y próxima, por tanto, al paradigma «tradicional artesanal» (Zeichner 1983).

Entendemos que los dos aspectos citados son trascendentales, tanto para la formación del profesorado como para los propios formadores, por lo que centramos en ellos nuestra reflexión.
Los sujetos encuestados reconocen mayoritariamente que los alumnos de niveles obligatorios no superan los objetivos propuestos por la enseñanza de las Ciencias y que el bachillerato aporta al estudiante unos ligeros conocimientos teóricos memorísticos, desarrollando, en el mejor de los casos, la capacidad de adaptarse al sistema educativo actual y obtener los éxitos académicos perseguidos. Tampoco parece, a juicio de los consultados, que la enseñanza de las Ciencias promueva actitudes positivas hacia la Ciencia. Conviene mencionar aquí la apreciación señalada por algunos profesores en formación, en el sentido de que la figura del profesor, su carisma, su capacidad de conexión con el alumnado influyen en la promoción del interés científico; idea coherente, hasta cierto punto, con la de que «profesor, se nace».

Además, los profesores reconocen que la Enseñanza de las Ciencias ha de aumentar los ámbitos de sus objetivos y superar la exclusiva introducción de los referidos a conceptos, leyes y teorías. Prácticamente todos los colectivos destacan la conveniencia de acercar el conocimiento científico al ambiente cotidiano, a los intereses de los alumnos, etc. y de introducir los procesos de la Ciencia. Los profesores en activo son más críticos con relación a lo segundo, debido posiblemente a cierto grado de frustración que se produce al preparar actividades «prácticas» y observar que con ellas el alumno no siempre alcanza hábitos/destrezas científicas, conocimientos significativos, ni tan siquiera una actitud positiva hacia la Ciencia (Bastida de Ia Calle 1990). Sin embargo, para los profesores en formación y sin experiencia, las «prácticas» constituyen la piedra de toque de la enseñanza científica y la verdadera «asignatura pendiente» de la misma (Miguens y Garrett 1991).

Por otra parte, en este trabajo y en nuestra experiencia en la formación de docentes, hemos podido observar que el profesor, desencantos aparte, siente la necesidad de que

se produzca un cambio en las estrategias aplicadas a la enseñanza de las Ciencias (buscar fórmulas que hagan del aprendizaje científico un actividad motivadora, significativa...).

Todo lo expuesto parece indicar que los profesores poseen ideas críticas y realistas sobre la enseñanza de las Ciencias, demandando un cambio en Educación (Busto y Osoco 1990). A esto contraponemos sus opiniones sobre las vías que propiciarían la optimización de dicha enserianza. En este sentido los datos ofrecidos en este trabajo muestran que los docentes, en general, son poco conscientes de la influencia que tiene su formación en el exito de la enseñanza y demuestran explícitamente un alto grado de «influencia ambiental» (Furió y Gil 1989). $S i$ bien admiten que el profesor debe estar capacitado para realizar numerosas actividades (programación, evaluación...), consideran que la experiencia directa en el aula seguida de las lecturas personales son los sistemas de formación docente por excelencia, siendo la asistencia a cursos y la implicación en la investigación điđáctica escasamente estimados. Ante esta situación se nos plantean los siguientes interrogantes: ¿Son realmente próximos y significativos para el docente los cursos 
impartidos? ¿Creen los docentes que, al igual que ocurre en otras profesiones, la investigación en la enseñanza tiene una incidencia directa en el aula? La contestación a ambas preguntas implica directamente al Sistema de Formación del Profesorado, a los formadores y a la propia Investigación Didáctica.

Salvo excepciones, los currículos de formación de profesorado tienen un carácter sumativo de conocimientos "científicos» y "psicopedagógicos» sin que exista la necesarja conexión entre ellos (Calderhead 1986, MacDermott 1990). Tal planteamiento no siempre resulta útil y significativo para el docente, en la medida que no da respuesta a sus problemas didácticos concretos. La cuestión que surge ahora es: ¿cómo ha de ser tal formación? Nosotras apostamos por una formación ante todo significativa, útil y práctica, pero sin olvidar los aspectos teóricos que la fundamentan y, cómo no, la investigación en Didáctica de las Ciencias.

Consideramos que la Didáctica de las Ciencias tiene un papel trascendente en la Formación profesional docente. Esta Área de conocimiento ha de servir de elemento integrador entre aspectos científicos y psicopedagógicos (Dumas-Carré et al. 1990, Gil 1991, documento de trabajo) y ha de permitir fundamentar teóricamente, a la luz de la investigación, las actividades y funciones que el profesor de Ciencias tiene que realizar: $a$ ) seleccionar contenidos/objetivos y jerarquizarlos de acuerdo con las capacidades intelectuales y necesidades del alumnado; b) optar por estrategias concretas de aprendizaje; $c$ ) programar sistemas de evaluación adecuados; etc. Todo ello encierra difícultades y demanda un proceso de

\section{REFERENCIAS BIBLIOGRÁFICAS}

BASTIDA DE LA CALLE, M.F., RAMOS, F. y SOTO, I., 1990. Prácticas de laboratorio: ¿una inversión poco rentable?, Investigación en la Escuela, 11, pp. 77-91.

BRISCOE, C., 1991.The dynamic interactions among beliefs. A case study of teacher change, Sciene Education, 75(2), pp. 185-199.

BUSTO, J.L. y OSOCO, A., 1990. La reconversión pedagógica del profesorado, Cuadernos de Pedagogia, 183, pp. 66-69.

CALDERHEAD, J., 1986.La mejora de la práctica de clase: aplicaciones de la investigación sobre toma de decisiones en la formación del profesorado. Actas del l Congreso Internacional sobre Pensamiento de los Profesores y Toma de Decisiones (Servicio de Publicaciones de la Universidad de Sevilla). aprendizaje, resultando acientífico y poco rentable que el docente adquiera las capacidades citađas a través, exclusivamente, de procesos ensayo-error (paradigma tradicional-artesanal) y de lecturas más o menos dispersas.

Las estrategias empleadas en la formación docente deben promover el cambio didáctico (Gil 1991). Para ello es necesario considerar como paso inicial el que los profesores analicen, critiquen y evidencien la «docencia del sentido común (Gené y Gil 1987, Briscoe 1991) tan arraigada entre ellos, llegando a admitir que la formación docente es uno de los factores centrales que influyen en la calidad de la enseñanza.

No olvidamos tampoco aquí un aspecto tan importante como la investigación. El profesor dispone de un material de excepción (sus propios alumnos) para investigar y evaluar nuevos planteamientos didácticos. Consideramos, por tanto, que el docente debe pasar de ser un mero consumidor de la investigación didáctica para involucrarse en ella (Santos 1990), por lo que ésta debería contemplarse en la formación inicial/permanente del profesorado (Lawson et al. 1986, Penick y Yager 1986).

De lo expuesto, se podría deducir que nuestra opción en la formación del profesor de Ciencias es excesivamente teórica. Sin embargo tal punto debe ser claramente matizado. El hechode que hayamos criticado «la experiencia docente» como única vía de formación no significa que la desestimemos, pues consideramos su transcendencia, pero va a ser la fundamentación teórica la que haga realmente significativa tal experiencia (Zeichner 1983).

CALDERHEAD, J., 1989. Reflective teaching and teacher education, Teaching \& Teacher Education, 5(1), pp. 43-51.

DUMAS-CARRÉ, A., FURIÓ, C. y GARRET, R., 1990. Formación inicial del profesorado de Ciencias en Francia, Inglaterra y Gales y España. Análisis de la organización de los estudios y nuevas tendencias, Enseñanza de las Ciencias, 8(3), pp. 274-281.

FURIO, C. y GIL, D., 1989. La didáctica de las ciencias en Ia formación inicial de profesorado, una orientacion y un programa teóricamente fundamentados, Enseñanza de Las Ciencias, 7(2), pp. 257-265.

GENÉ, A. y GIt, D., 1987. Tres principios básicos en el diseño de la formación del profesorado, Antecha Pedagógica,18, pp. 28-30. 
GIL, D., 1991. ¿Qú́ debemos de saber y saber hacer los profesores de Ciencias? Intento de síntesis de las aportaciones de la investigación didáctica, Enseñanza de la Ciencias, 9(1), pp. 69-77.

GIL, D., 1991. La formación inicial del profesorado de educación secundaria. Análisis crítico y propuestas de remodelación. (Documento de trabajo).

GIL, D., CARRASCOSA, J., FURIÓ, C. y MARTÍNEZTORREGROSA, J., 1991. La enseñanza de las ciencias para la educación secundaria. (Horsorí e ICE de la Universitat de Barcelona).

GUTIÉRREZ, R., MARCO, B., OLIVARES, E. y SERRANO, $T ., 1990$. Enseñanza de las ciencias en la educación intermedia. (Rialp: Madrid).

JIMÉNEZ, M.P. y OTERO, J., 1990. Laciencia como construcción social, Cuadernos de Pedagogía, 180, pp. 20-22.

LAWSON, A.E, COSTENSON, K. y CISNEROS, R., 1986. A summary of research in science education, Science Education, $70(3)$, pp. 191-346.

MATTHEWS, M.R., 1990. History, philosophy and science teaching: a rapprochement, Studies in Science Education, 18 , pp. $25-51$.

McDERMOTT, L., 1990. A perspective on teacher preparation in physics and other science: The need for special science courses for teachers, American Journal of Physics, 58(8), pp. 734-742.

MIGUENS, M. y GARRETT, R.M., 1991. Prácticas en la enseñanza de las ciencias. Problemas y posibilidades, Enseñanza de la Ciencias, 9(2), pp. 229-236.

OTERO, J., 1989. La producción y la comprensión de Ia ciencia: Ia elaboración en el aprendizaje de la ciencia escolar, Enseñanza de las Ciencias, 7(3), pp. 223-228.

PENICK,J.E. y YAGER, R.E., 1986. Trends in science education: some observations of exemplary programmes in the United States, European Journal of Sciencie Education, 8(1), pp. 1-8.

SALTIEL, E. y VIENNOT, L., 1985. ¿Qué aprendemos de las semejanzas entre las ideas históricas y el razonamiento espontáneo de los estudiantes?, Enseñanza de las Ciencias, 3(2), pp. 137-144.

SANTOS, M.A., 1990. Del diseño y desarrollo curricular como marco de la formación del profesorado, Investigación en la Escuela, 10, pp. 23-33.

SOLBES, J. y VILCHES, A., 1989. Interacciones Ciencia/ Técnica/Sociedad: Un instrumento de cambio actitucional, Enseñanza de las Ciencias, $7(1)$, pp. 14-20.

TOBIN, K. y ESPINET, M., 1989. Impediments to change: applications of coaching in high school science teaching, Journal of research in Science Teaching, 26(2), pp. 105120.

VERMA, J. y BEARD, R.M., 1981. What is educational research (Gower: London).

ZEICHNER, K.M., 1983. Alternative paradigms of teacher education, Journal of teacher Education, 34(3), pp. 3-9.
1. Valora de uno a cinco tu interés por la Enseñanza de las Ciencias.

2. Valora de uno a cinco los objetivos que la enseñanza de las Ciencias debe alcanzar al final de la enseñanza obligatoria.

a) Conocer teorías y conceptos científicos.

b) Utilizar conceptos científicos en contextos cotidianos.

c) Valorar la incidencia positiva y negativa que tiene y ha tenido la Ciencia en las sociedades humanas.

d) Conocer la metodología científica y saber utilizarla en Ia resolución de problemas concretos.

e) Conocer la influencia que la sociedad y la comunidad científica ha tenido en la aceptación de determinadas teorías y descubrimientos, es decir, asociar el descubrimiento científico al momento histórico.

3. ¿Cuáles crees que son los objetivos que un estudiante llega a alcanzar después del bachillerato actual?

4. ¿Consideras que actualmente la enseñanza de las Ciencias permite a los jovenes interesarse por la Ciencia?
5. Crees que la enseñanza de las Ciencias permite alcanzar los objetivos que a tu juicio son más interesantes?

6. ¿Cuál sería, a tu juticio, el camino para alcanzar los citados objetivos?

7. El profesor de Ciencias debe ser capaz de:

a) Programar y llevar a cabo con sus alumnos experiencias de campo y laboratorio.

b) Seleccionar los contenidos adecuados.

c) Elegir los textos y manuales que ha de recomendar a sus alumnos.

d) Detectar los errores más significativos que tienen sus alumnos.

e) Valorar Ios intereses de sus alumnos y actuar en consecuencia.

f) Comprender las orientaciones pedagógico-didácticas elaboradas por las autoridades editcativas y aplicarlas al contexto de su clase.

g) Llevar a cabo planteamientos didácticos concretos. 
h) Evaluar el aprendizaje de los alumnos.

i) Evaluar en su conjunto las programaciones realizadas por él.

j) Analizar críticamente las nuevas tendencias en la enseñanza de las Ciencias.

8. Valora de uno a cinco en qué basas o basarías tu formacion como profesor de Ciencias

a) Lecturas de temas científicos.

Nota:

En Ia encuesta propuesta a profesores y a alumnos del CAP se ha desglosado la opción $d$ en las siguientes:

di) Asistencia a cursos de formación psicopedagógica.

d2) Asistencia a cursos de Didáctica de las Ciencias. b) Lecturas de temas didácticos específicos de Ciencias.

c) Observación cotidiana de tu actuación en el aula y de la respuesta que a ella dan los alumnos.

d) Asistencia a cursos especializados de temas educativos.

e) Participación activa en Ia investigación educativa.

f) Asistencia a congresos. 